\title{
Equality in Obstetrical Care: Racial/Ethnic Variation in Group B Streptococcus Screening
}

\author{
Allison S. Bryant · Yvonne W. Cheng • \\ Aaron B. Caughey
}

Published online: 19 September 2010

(c) The Author(s) 2010. This article is published with open access at Springerlink.com

\begin{abstract}
The objective of this article is to determine whether racial/ethnic disparities exist in screening for group B streptococcus (GBS) colonization among pregnant women. A retrospective cohort study of deliveries at a single institution was conducted. The primary outcome was the availability of GBS culture data at the time of delivery; the primary predictor was maternal race/ethnicity. Analyses were stratified by the time periods before and after the CDC recommendations for universal screening for GBS. Among 16,333 deliveries, $60.4 \%$ of the population was screened for GBS but screening rates varied markedly by year of delivery. Black women had a lower odds of having available GBS data (AOR $0.81[0.69,0.95]$ ) but this disparity was limited to the period of time before universal screening was recommended. Prior to the recommendation for universal screening for GBS, racial/ethnic disparities existed in rates of screening among pregnant women delivering at term. These differences were reduced after 2002, suggesting that uniform policies regarding obstetrical care may be effective in eliminating disparities in obstetrical care and outcomes.
\end{abstract}

Keywords GBS screening - Disparities - Quality of care

\footnotetext{
A. S. Bryant ( ()

Department of Obstetrics and Gynecology, Massachusetts General Hospital, 55 Fruit Street, Boston, MA 02114, USA e-mail: abryant@partners.org

Y. W. Cheng

Department of Obstetrics, Gynecology and Reproductive Sciences, University of California, San Francisco, San Francisco, CA, USA

\author{
A. B. Caughey \\ Department of Obstetrics and Gynecology, Oregon Health \& \\ Sciences University, Portland, OR, USA
}

\section{Introduction}

Wide disparities in health outcomes have been described for racial and ethnic minorities in the United States. The Institute of Medicine, charged with examining disparate health outcomes, found that a large body of research supports inequities in health care as possible contributors to inequities in health [1]. Though disparities in obstetrical outcomes have been well documented [2-4], differential obstetrical care by maternal race/ethnicity has been less well studied [5].

The prevention of early onset group B streptococcal (GBS) infection in neonates has become a major public health effort. The 1990s saw a significant decline in cases of early GBS disease, from 2 to 3 cases per 1,000 live births to 0.5 cases per 1,000 live births, thought largely due to more widespread use of intrapartum antibiotic prophylaxis (IAP) [6, 7]. In 1996, the Centers for Disease Control and Prevention (CDC) issued guidelines for the prevention of perinatal GBS disease, and allowed obstetrical providers to choose between two strategies for maternal risk stratification [8]. In one (risk factor-based), women presenting in labor were evaluated for risk factors for early onset GBS disease (preterm gestation, fever in labor, prolonged rupture of membranes, for example) and treated if criteria for risk were met. In the other strategy (screening-based), recto-vaginal cultures for GBS were collected between 35 and 37 weeks gestation, and women with positive cultures were treated with IAP in labor. In 2002, the CDC released new guidelines, reflective of surveillance data collected since the 1996 release, which compared the effectiveness of the two screening strategies [9]. The new recommendations included guidelines for universal GBS screening, with the risk-factor based approach deemed to be a substandard strategy. 
The demonstration of racial/ethnic disparities in rates of GBS screening would provide evidence of inconsistencies in the quality of care delivered to obstetrical patients and would serve as a concrete area for quality improvement efforts on the part of health care providers and systems. The documentation of such inequities generally provides a first step towards ameliorating them. To date, few metrics of quality of obstetric care have been identified and used to assess quality, equality or adequacy of care. The finding of disparities in a process of care measure such as GBS screening may be causally related to disparities in neonatal outcomes. In the current study, we sought to test our hypothesis that racial and ethnic minority women, in particular, Black women, would be less likely to be screened for GBS than White women, both during the time period in which screening or risk-factor based strategies were endorsed by CDC (1996-2002), as well as during the period of recommendation for universal screening (2002 and beyond). We also tested the hypothesis that disparities by race/ethnicity would exist irrespective of insurance status (i.e., publicly vs. privately insured), and that among those women screened, rates of GBS carriage were higher among Black women, in particular.

\section{Materials and Methods}

We conducted a retrospective cohort study of women delivering at the University of California, San Francisco (UCSF) between 1996 and 2008. The primary outcome for this study was availability of a recto-vaginal GBS culture result at delivery as a measure of having had screening for GBS performed; the primary predictor was self-reported maternal race/ethnicity. In addition, data on maternal sociodemographic and clinical characteristics were evaluated as covariates. The UCSF Department of Obstetrics, Gynecology \& Reproductive Sciences maintains a perinatal database of all deliveries occurring at UCSF Moffitt-Long Hospital. This is a prospectively collected, research-quality obstetric database. Each woman's prenatal and inpatient medical record is abstracted by trained research personnel and entered into this database.

Women who delivered at or beyond 37 completed weeks of gestation were included in these analyses, as recommendations for the screening strategy call for cultures to be conducted between 35 and 37 weeks of pregnancy. Women transported to our institution for delivery were excluded from analyses, as were women with no information on maternal race/ethnicity. We conducted bivariate analyses, using chi-squared tests, t-tests and logistic regression to assess the association between availability of GBS culture and each maternal race/ethnicity category, as well as for several other potential confounding factors such as maternal age, parity, number of prenatal care visits, history of a preterm delivery and insurance status. Those variables that were found to be statistically significantly associated (at the $P<0.10$ level) with availability of GBS culture were retained in multivariable logistic regression models which predicted GBS culture availability as a function of maternal race/ethnicity. These models were also adjusted for gestational age at delivery and year of delivery. We stratified analyses by time period (1996-2001 and 2002-2008), and also included a test for trend by year of delivery. We tested our hypothesis that disparities in GBS screening are not modified by maternal insurance status by testing an interaction between race/ethnicity and insurance status.

To demonstrate consistency with findings in other populations, we sought to confirm that rates of GBS carriage are higher among racial and ethnic minorities in our population by creating multivariable logistic models of GBS carriage among women for whom culture data were available. All analyses were conducted using the Stata 9 statistical package (StataCorp, College Station, TX). This study received institutional approval from the Committee on Human Research at the University of California, San Francisco.

\section{Results}

After exclusion of 603 women with missing data on race/ ethnicity, there were 16,333 women who met study inclusion criteria. Overall, GBS culture data were present at delivery in $60.4 \%$ of all patients. This varied greatly by year of delivery: for those women delivering between 1996 and 2001 (before the CDC recommendation for universal screening), the proportion of available cultures was $26.9 \%$. Between 2002 and 2008, the proportion of available culture data was $86.2 \%$. In 2008, the proportion was $98.5 \%$. Characteristics of the study population are shown in Table 1. In unadjusted analyses, Asian (odds ratio (OR) 0.86 [95\% CI 0.80, 0.94]) and Black women OR 0.73 $[0.66,0.80]$ were less likely to have GBS results available than were white women. Known GBS status at delivery was also associated with older maternal age and private insurance. In multivariable regression modeling, also presented in Table 1, Black race remained an independent predictor of absence of a GBS result at delivery (adjusted odd ratio (AOR) 0.80 [0.69, 0.94]). Models were adjusted for parity, public vs. private insurance, number of prenatal visits, gestational age, prior preterm delivery, and year of delivery.

When we stratified the population by year of delivery (1996-2001 vs. 2002-2008; Table 2), Black women remained statistically significantly at lower odds of having 
Table 1 Socio-demographic and clinical characteristics of the study population, $N=16,333$

$\begin{array}{ll}\% \text { population } & \% \text { with } \\ \text { or }(\text { mean } \pm \mathrm{SD}) & \begin{array}{l}\text { available GBS } \\ \text { culture or } \\ (\text { mean } \pm \mathrm{SD})\end{array}\end{array}$

\begin{tabular}{llll}
\hline Race/ethnicity & & & $<0.001$ \\
Asian & 17.3 & 58.9 & \\
Black & 12.8 & 54.8 & \\
Latina & 11.4 & 62.1 & \\
White & 45.9 & 62.4 & \\
Other & 12.6 & 59.0 & \\
Age & $(30.2 \pm 6.2)$ & $(30.7 \pm 6.1)$ & $<0.001$ \\
Parity & & & 0.07 \\
Nulliparous & 54.9 & 61.0 & \\
Multiparous & 45.1 & 59.6 & \\
Insurance & & & $<0.001$ \\
Private & 75.6 & 62.0 & \\
Public & 24.4 & 55.3 & $<0.001$ \\
Number of visits & $(7.9 \pm 3.2)$ & $(7.5 \pm 3.2)$ & $<0.001$ \\
Previous preterm delivery & 2.1 & 50.3 & \\
Year of delivery & & & \\
1996-2001 & 43.5 & 26.9 & \\
2002-2008 & 56.5 & 86.2 & \\
\hline
\end{tabular}

GBS culture data available at delivery in the earlier time period (AOR $0.79[0.65,0.97])$ but this lower odds did not reach statistical significance in the later time period (AOR $0.85[0.65,1.12])$. During 1996-2001, Asian women were also less likely to have known GBS status (AOR 0.75 $[0.63,0.88])$, but not during 2002-2008. In the earlier time period public health insurance coverage was associated with a lower odds of GBS status availability (AOR 0.83 $[0.71,0.98])$, but not in the later time period. No significant interactions were noted between race/ethnicity and insurance status during either time period.

A test for trend shows a significant increase in the odds of available GBS data at delivery in more recent years $(P<0.001)$. Relative to the year 2002 , the odds of having a GBS culture available in 1996 was 0.61 [0.51, 0.73], and was 140.3 [76.2, 258.5] in 2008. Figure 1 demonstrates the proportion of women with available GBS data by race/ ethnicity and year of delivery. Prior to 2002, women of racial/ethnic minorities were less likely, on average, to have GBS screening completed by delivery, while after 2002, the differences are less pronounced.

When we explored the rates of GBS-positive culture among women with culture data available at delivery, women of all minority groups were more likely to have GBS detected on rectovaginal culture than were white women (AOR for Asians 1.22 [1.07, 1.40], Blacks 1.98
[1.68, 2.35], Latinas 1.32 [1.13, 1.55] and other 1.31 [1.12, 1.53]; Table 3).

\section{Comment}

In our population of women with term deliveries, Black women were significantly less likely to be screened for GBS than were White women. These findings were most significant during the time period when the CDC allowed for a risk-factor based or screening-based approach to the reduction of early-onset GBS disease (EOD). Following their 2002 recommendations for universal screening for GBS, rates of screening were similar for women of different race/ethnicities. During the same time period, there was a dramatic increase in overall screening rates for GBS, as has been documented elsewhere [10].

Disparities in recommended screening for GBS are of concern, especially in light of the recent release of surveillance data for EOD in the United States between 2000 and 2006. It is well known that Black race is a risk factor for EOD $[6,11,12]$. The most recent data demonstrate that the sole group in which the incidence of EOD is on the rise is Black term infants, a group which saw an increase in incidence from 0.33 cases per 1,000 live births to 0.70 cases per 1,000 live births from 2003 to 2006 [13]. Inequities in quality of care may contribute to inequities in neonatal outcomes.

A prior study in North Carolina in 2002-2003 showed that Hispanic women and women receiving care in a hospital clinic or health department were less likely to be screened for GBS [14]. This study relied on maternal selfreport; our study has as a strength our ability to have objective evidence of GBS status availability. Another investigation of predictors of screening for perinatal infections in 2000-2001 revealed no differences by race/ethnicity or insurance status with regards to universally-recommended screenings for infections such as hepatitis B, syphilis and rubella, but did show some variation by both race/ethnicity and insurance for infections in which recommendations at the time were based on risk factors, such as GBS, hepatitis $\mathrm{C}$ and varicella [15]. The authors suggest that the systems of care in which minority and poor women may be enrolled may have different practice standards, reflective of provider and patient awareness, as well as reimbursement rates of insurers. Our finding that race/ethnicity and insurance predicted GBS screening in the era prior to the recommendation for universal screening, but not after, would support this notion. The institution of uniform practice standards and the reduction in permissive variability in care may be effective strategies to reduce disparities in health care and outcomes. We also confirm a higher risk among Black women of carriage of GBS among those women who 
Table 2 Unadjusted and adjusted odds of available GBS culture, in all years and stratified by year of delivery

\begin{tabular}{|c|c|c|c|c|c|c|}
\hline & $\begin{array}{l}\text { Unadjusted OR for } \\
\text { available GBS } \\
\text { culture }(95 \% \mathrm{CI}) \text { : } \\
\text { all years }\end{array}$ & $\begin{array}{l}\text { Adjusted } \mathrm{OR}^{\mathrm{a}} \text { for } \\
\text { available } \mathrm{GBS} \\
\text { culture }(95 \% \mathrm{CI}) \text { : } \\
\text { all years }\end{array}$ & $\begin{array}{l}\text { Unadjusted OR for } \\
\text { available GBS } \\
\text { culture }(95 \% \text { CI): } \\
\text { 1996-2001 }\end{array}$ & $\begin{array}{l}\text { Adjusted } \mathrm{OR}^{\mathrm{a}} \text { for } \\
\text { available GBS } \\
\text { culture }(95 \% \mathrm{CI}) \text { : } \\
\text { 1996-2001 }\end{array}$ & $\begin{array}{l}\text { Unadjusted OR for } \\
\text { available GBS } \\
\text { culture }(95 \% \text { CI): } \\
\text { 2002-2008 }\end{array}$ & $\begin{array}{l}\text { Adjusted } \mathrm{OR}^{\mathrm{a}} \text { for } \\
\text { available GBS } \\
\text { culture }(95 \% \mathrm{CI}) \text { : } \\
\text { 2002-2008 }\end{array}$ \\
\hline \multicolumn{7}{|l|}{ Race/ethnicity } \\
\hline Asian & $0.86(0.80,0.94)$ & $0.89(0.78,1.01)$ & $0.79(0.68,0.92)$ & $0.75\left(\begin{array}{lll}0.63 & 0.88\end{array}\right)$ & $1.09(0.92,1.30)$ & $1.18(0.95,1.47)$ \\
\hline Black & $0.73(0.66,0.80)$ & $0.81(0.69,0.95)$ & $0.77(0.65,0.91)$ & $0.79(0.65,0.97)$ & $0.86(0.72,1.04)$ & $0.85(0.65,1.12)$ \\
\hline Latina & $0.98(0.89,1.09)$ & $0.92(0.78,1.08)$ & $1.01(0.85,1.21)$ & $1.01(0.83,1.22)$ & $1.10(0.92,1.30)$ & $0.80(0.611 .03)$ \\
\hline White & 1.00 & 1.00 & 1.00 & 1.00 & 1.00 & 1.00 \\
\hline Other & $0.86(0.78,0.95)$ & $0.88(0.76,1.02)$ & $0.84(0.71,1.00)$ & $0.80(0.66,0.96)$ & $1.16(0.95,1.42)$ & $1.05(0.81,1.36)$ \\
\hline Age & $1.03(1.02,1.03)$ & $1.00(0.99,1.01)$ & $1.01(1.00,1.01)$ & $1.00(0.99,1.01)$ & $1.01(1.00,1.02)$ & $0.99(0.98,1.00)$ \\
\hline \multicolumn{7}{|l|}{ Parity } \\
\hline Nulliparous & $1.06(1.00,1.12)$ & See footnote & $0.95(0.86,1.06)$ & See footnote & $1.28(1.14,1.44)$ & See footnote \\
\hline Multiparous & 1.00 & & 1.00 & & 1.00 & \\
\hline \multicolumn{7}{|l|}{ Insurance } \\
\hline Private & 1.00 & 1.00 & 1.00 & 1.00 & 1.00 & 1.00 \\
\hline Public & $0.75(0.71,0.82)$ & $0.87(0.77,0.99)$ & $0.86(0.77,0.97)$ & $0.83(0.71,0.98)$ & $0.93(0.81,1.08)$ & $0.92(0.74,1.13)$ \\
\hline $\begin{array}{l}\text { Number of } \\
\text { visits }\end{array}$ & $0.91(0.90,0.92)$ & See footnote & $0.98(0.97,1.00)$ & See footnote & $1.04(1.02,1.06)$ & See footnote \\
\hline $\begin{array}{l}\text { Previous } \\
\text { preterm } \\
\text { delivery }\end{array}$ & $0.69(0.53,0.82)$ & $1.45(1.09,1.94)$ & $1.44(1.09,1.92)$ & $1.50(1.10,2.04)$ & $0.62(0.40,0.98)$ & $0.72(0.40,1.30)$ \\
\hline \multicolumn{7}{|c|}{ Year of delivery } \\
\hline 1996-2001 & 1.00 & See footnote & & & & \\
\hline 2002-2008 & $16.9(15.6,18.3)$ & & & & & \\
\hline
\end{tabular}

${ }^{a}$ Adjusted for other covariates presented, in addition to parity (as a continuous variable), gestational age, number of prenatal visits (as a categorical variable) and year of delivery

Values in italics have $P<0.05$

\section{Availability of GBS culture data at delivery (\%)}

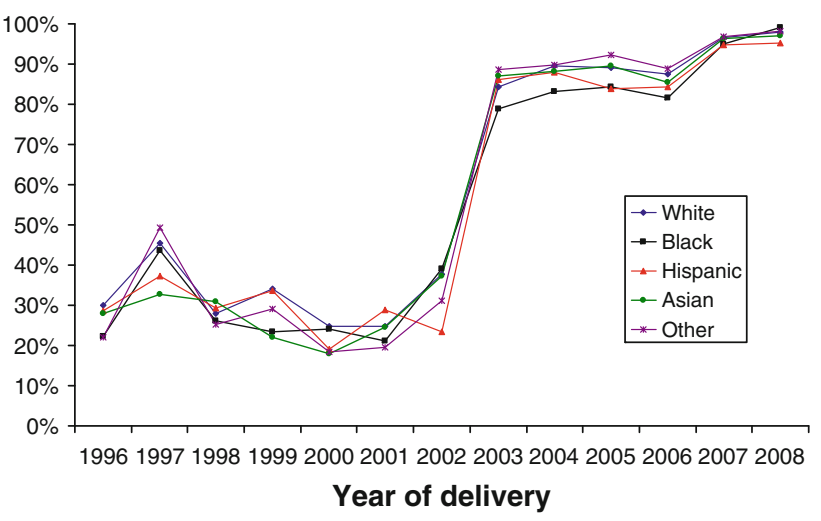

Fig. 1 Proportion of women with available GBS culture data, by year of delivery and maternal race/ethnicity

were screened during pregnancy, consistent with other reports [16, 17], and reiterating the need for adherence to best practices for screening in all populations.

Our study benefits from its conduct in a single delivery center and from inclusion only of patients with term
Table 3 Risk of presence of GBS on rectovaginal culture by race/ ethnicity, among women with known GBS status

\begin{tabular}{lll}
\hline Race/ethnicity & GBS+/number screened $(\%)$ & Adjusted $\mathrm{OR}^{\mathrm{a}}$ \\
\hline Asian & $415 / 1,733(24.1)$ & $1.22(1.07,1.40)$ \\
Black & $432 / 1,192(36.2)$ & $1.98(1.68,2.35)$ \\
Latina & $321 / 1,201(26.7)$ & $1.32(1.13,1.55)$ \\
White & $990 / 4,836(20.5)$ & 1.00 \\
Other & $311 / 1,255(24.8)$ & $1.31(1.12,1.53)$ \\
\hline
\end{tabular}

a Adjusted for maternal age, parity, gestational age, number of prenatal visits, insurance, year of delivery and prior preterm delivery

Values in italics have $P<0.05$

deliveries, not transported to our institution from other centers. We therefore believe that our ascertainment of availability of GBS data is quite good, though we are not able to validate the accuracy of the perinatal database using primary microbiology laboratory records. While there is a possibility of differential documentation of GBS status in the medical chart by race/ethnicity or practice, we believe this to be minimal. Our ability to capture deliveries from the entire period from the $1996 \mathrm{CDC}$ recommendations for 
screening vs. risk factor based protocols, through their mandate for universal screening and into the present day also represents a strength. We do not believe the association between race/ethnicity and availability of GBS results to be confounded by the natures of the practices in which patients are seen; in our institution, insurance status is a close proxy for obstetrical practice, and we have adjusted for the former in our analyses.

Our study is not without limitations, however. Our outcome is derived from review of the medical record, and not from laboratory data. While in some ways this limits our ability to comment on "true availability" of culture data, our outcome definition lies closest to the information available to the providers at the time prenatal and intrapartum care was rendered. Other studies have documented that, despite high rates of overall screening since 2002, screening may not occur at the appropriate time (e.g., prior to 35 weeks, then not repeated) $[18,19]$; we did not look specifically at this phenomenon. We are also unable to comment on rates on intrapartum antibiotic prophylaxis among women with risk factors or positive screens for GBS in this population; this rate represents another potential measure of obstetrical care quality. And lastly, while it is likely that Black women in this population were somewhat more likely to have had GBS bacteriuria or a prior neonate affected by invasive GBS disease, and thus appropriately not screened by recto-vaginal culture in the index pregnancy, we do not believe that the magnitude of the racial/ ethnic differences in these conditions explains the extent of the absolute difference in screening rates that we witnessed in the early period.

Disparities in quality of care exist throughout health care. Racial and ethnic minorities are less likely to receive recommended immunizations, less likely to be screened for colorectal cancer and less likely to receive weight management counseling if obese [20]. It is therefore not surprising that disparities in quality of obstetrical care exist as well. In our field, however, we have relatively few agreedupon metrics of quality care [21]. Screening for GBS, while not a measure used by groups such as the Agency for Healthcare Research and Quality when reporting quality of care, is clearly a key component of antenatal care, with relevance to neonatal outcomes. Disparities in this procedure should raise a warning that similar disparities likely exist in other areas of obstetric care that allow for similar variability in management styles as seen in the pre-2002 era of GBS screening. While other process measures are perhaps less easily measured, they may be no less important to maternal and infant outcomes. Increasing awareness of obstetrical disparities will hopefully serve to eliminate them. Clinical, research and health policy endeavors should have as a goal ensuring that patients, providers and systems of care all contribute positively to equitable processes of care and health outcomes.

Open Access This article is distributed under the terms of the Creative Commons Attribution Noncommercial License which permits any noncommercial use, distribution, and reproduction in any medium, provided the original author(s) and source are credited.

\section{References}

1. Smedley, B. D., Stith, A. Y., \& Nelson, A. R. (2003). Unequal treatment: Confronting racial and ethnic disparities in health care. Washington, DC: Institute of Medicine.

2. Kramer, M. S., Goulet, L., Lydon, J., Seguin, L., McNamara, H., Dassa, C., et al. (2001). Socio-economic disparities in preterm birth: Causal pathways and mechanisms. Paediatric and Perinatal Epidemiology, 15(suppl 2), 104-123.

3. Lu, M. C., \& Chen, B. (2004). Racial and ethnic disparities in preterm birth: The role of stressful life events. American Journal of Obstetrics and Gynecology, 191(3), 691-699.

4. Schoendorf, K., Hogue, C., Kleinman, J., \& Rowley, D. (1992). Mortality among infants of black as compared with white college-educated parents. New England Journal of Medicine, 326(23), 1522-1526.

5. Bryant, A., Worjoloh, A., Caughey, A., \& Washingon, A. (2009). Racial/ethnic disparities in obstetrical outcomes and care: Prevalence and determinants. American Journal of Obstetrics and Gynecology, 202(4), 335-343.

6. Phares, C. R., Lynfield, R., Farley, M. M., Mohle-Boetani, J., Harrison, L. H., Petit, S., et al. (2008). Epidemiology of invasive group B streptococcal disease in the United States, 1999-2005. JAMA, 299(17), 2056-2065.

7. Schrag, S. J., Zywicki, S., Farley, M. M., Reingold, A. L., Harrison, L. H., Lefkowitz, L. B., et al. (2000). Group B streptococcal disease in the era of intrapartum antibiotic prophylaxis. New England Journal of Medicine, 342(1), 15-20.

8. CDC. (1996). Prevention of perinatal group B streptococcal disease: A public health perspective. MMWR. CDC Surveillance Summaries, 45(RR-7), 1-24.

9. Schrag, S., Gorwitz, R., Fultz-Butts, K., \& Schuchat, A. (2002). Prevention of perinatal group B streptococcal disease. Revised guidelines from CDC. MMWR. Recommendations and Reports, 51(RR-11), 1-22.

10. Van Dyke, M. K., Phares, C. R., Lynfield, R., Thomas, A. R., Arnold, K. E., Craig, A. S., et al. (2009). Evaluation of universal antenatal screening for group B streptococcus. New England Journal of Medicine, 360(25), 2626-2636.

11. Centers for Disease Control and Prevention (CDC). (2007). Perinatal group B streptococcal disease after universal screening recommendations-United States, 2003-2005. MMWR. CDC Surveillance Summaries, 56(28), 701-705.

12. Schuchat, A., Oxtoby, M., Cochi, S., Sikes, R. K., Hightower, A., Plikaytis, B., et al. (1990). Population-based risk factors for neonatal group B streptococcal disease: Results of a cohort study in metropolitan Atlanta. Journal of Infectious Diseases, 162(3), 672-677.

13. Centers for Disease Control and Prevention (CDC). (2009). Trends in perinatal group B streptococcal disease-United States, 2000-2006. MMWR. Morbidity and Mortality Weekly Report, 58(5), 109-112.

14. Avery, M., Brown, H. W., Schrag, S., \& Phil, D. (2005). Disparities in universal prenatal screening for group B 
streptococcus-North Carolina, 2002-2003. Morbidity and Mortality Weekly Report, 54(28), 700-703.

15. Jessop, A. B., Watson, B., Mazar, R., \& Andrel, J. (2005). Assessment of screening, treatment, and prevention of perinatal infections in the Philadelphia birth cohort. American Journal of Medical Quality, 20(5), 253-261.

16. Stapleton, R. D., Kahn, J. M., Evans, L. E., Critchlow, C. W., \& Gardella, C. M. (2005). Risk factors for group B streptococcal genitourinary tract colonization in pregnant women. Obstetrics and Gynecology, 106(6), 1246-1252.

17. Taylor, J. K., Hall, R. W., \& Dupre, A. R. (2002). The incidence of group B streptococcus in the vaginal tracts of pregnant women in central Alabama. Clinical Laboratory Science, 15(1), 16-17.
18. Rodriguez, E., Raker, C. A., Paglia, M. J., \& Anderson, B. L. (2010). Compliance with group B streptococcus testing prior to labor and delivery. American Journal of Perinatology, 27(6), 475-479.

19. Goins, W. P., Talbot, T. R., Schaffner, W., Edwards, K. M., Craig, A. S., Schrag, S. J., et al. (2010). Adherence to perinatal group B streptococcal prevention guidelines. Obstetrics and Gynecology, 115(6), 1217-1224.

20. AHRQ. (2003). National healthcare disparities report. Rockville, MD: AHRQ

21. Bennett, T., \& Adams, M. (2002). Safe motherhood in the United States: Challenges for surveillance. Maternal and Child Health Journal, 6(4), 221-226. 